\title{
Plasmonic Gold Nanoparticles Stain Hydrogels for the Portable and High-Throughput Monitoring of Mercury Ions
}

Xiao Juan Du, ${ }^{\dagger}$ Yang Chen, ${ }^{\dagger}+$ Ling Yun Qin, ${ }^{\dagger}$ Hong Qun Luo, ${ }^{\dagger}$ Nian Bing Li, ${ }^{\dagger} *$ and Bang $\operatorname{Lin} \mathrm{Li}^{\dagger} *$

† Key Laboratory of Luminescence Analysis and Molecular Sensing (Southwest University), Ministry of Education, School of Chemistry and Chemical Engineering, Southwest University, Chongqing 400715, P. R. China.

$\$$ School of Architecture and Urban Planning, Chongqing University, Chongqing 400030, P. R. China.

Corresponding authors: B. L. Li (hemlibl@swu.edu.cn); N. B. Li (linb@swu.edu.cn)

This PDF file includes seven figures, one table, and eight pages:

Figure S1 (Page S2)

Figure S2 (Page S2)

Figure S3 (Page S3)

Figure S4 (Page S3)

Figure S5 (Page S4)

Figure S6 (Page S4)

Figure S7 (Page S5)

Table S1 (Pages S6 and S7)

Supporting References (Pages S7 and S8) 


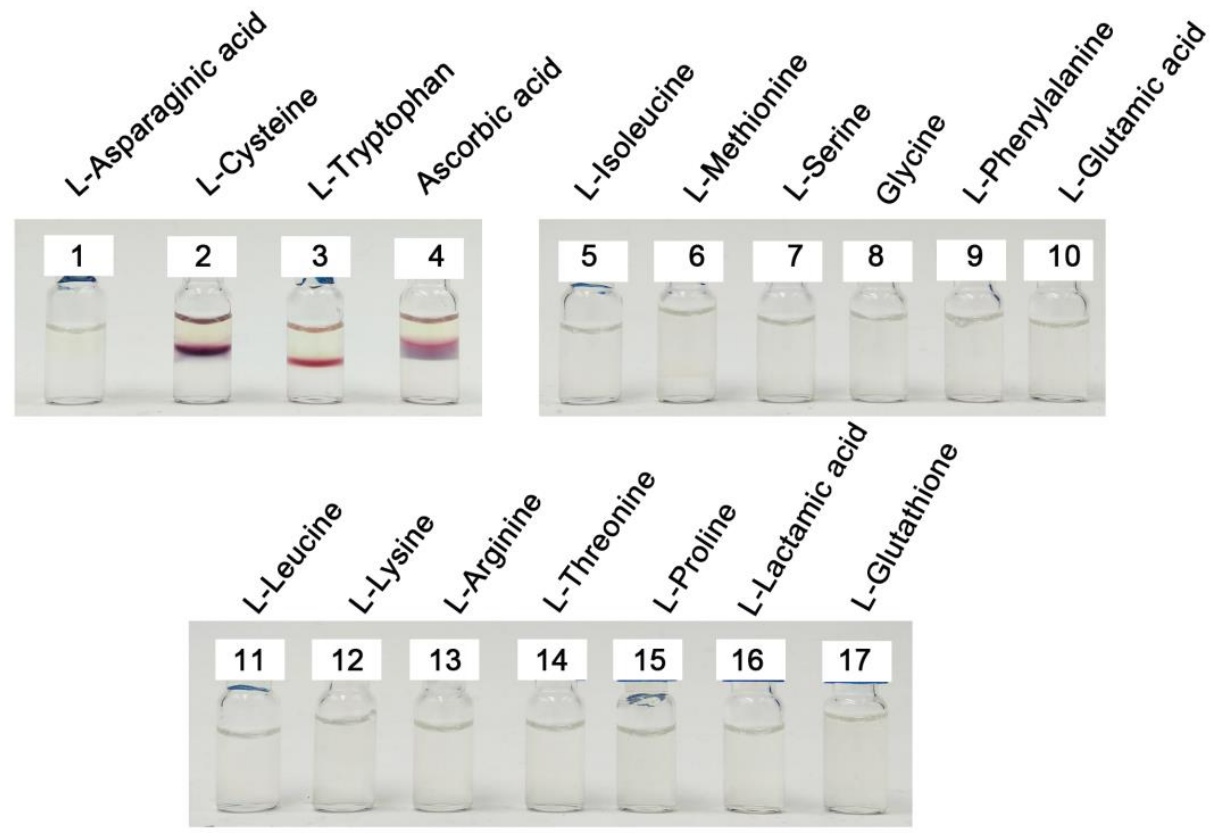

Figure S1. The photos of $\mathrm{HAuCl}_{4}$-incubating agarose hydrogels doped with diverse kinds of amino acids, ascorbic acid, and L-glutathione. In addition to L-cysteine, similar allochroic phenomenon could be observed on $\mathrm{HAuCl}_{4}$-incubating L-tryptophan-doped agarose hydrogels and ascorbic acid-doped agarose hydrogels. The concentrations of doped compounds and $\mathrm{HAuCl}_{4}$ are $0.1 \mathrm{mM}$, and $2.0 \mathrm{mM}$, respectively.

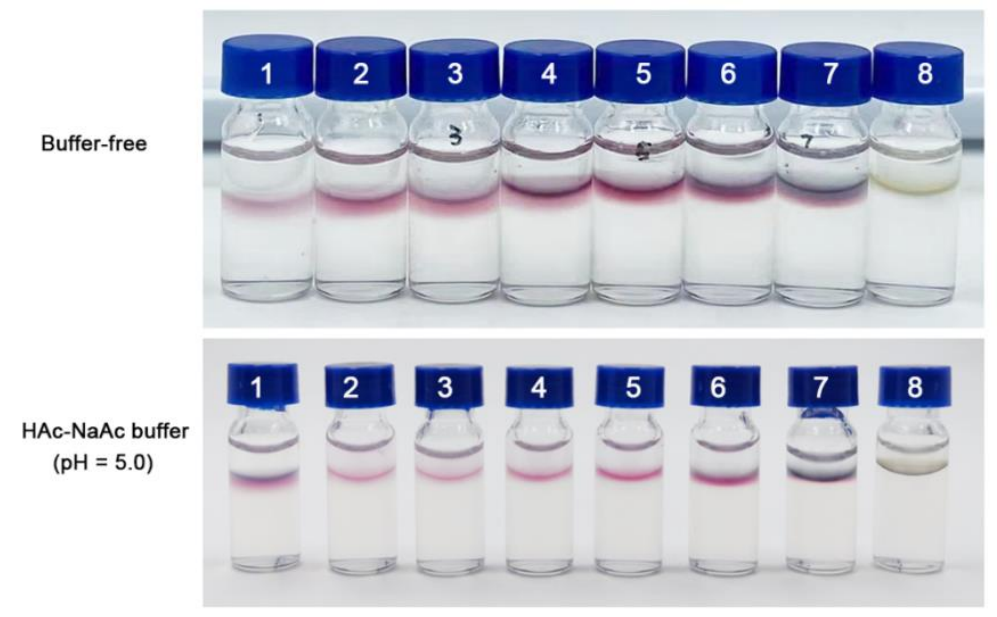

Figure S2. The Au-staining C-AGH treated with the various concentrations of $\mathrm{Hg}^{2+}$ ions in the absence and presence of buffer solutions $(\mathrm{pH}=5.0)$. The concentrations of $\mathrm{Hg}^{2+}$ from sample 1 to 8: 0.00, 0.01, 0.02, 0.05, 0.20, 0.50, 2.00, $5.00 \mathrm{mM}$. 
a

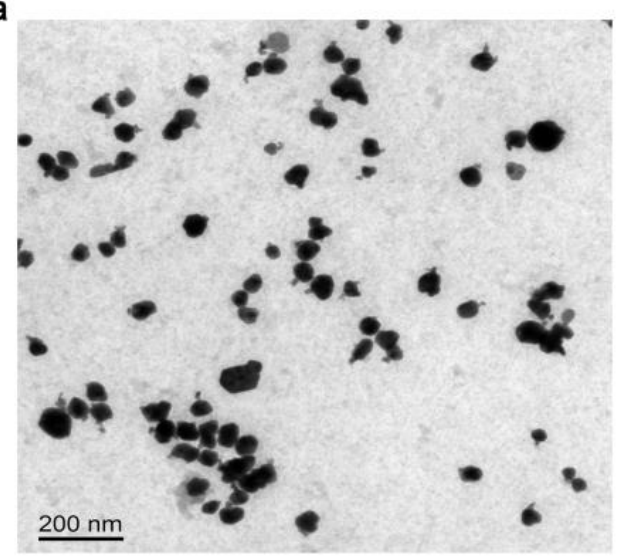

b

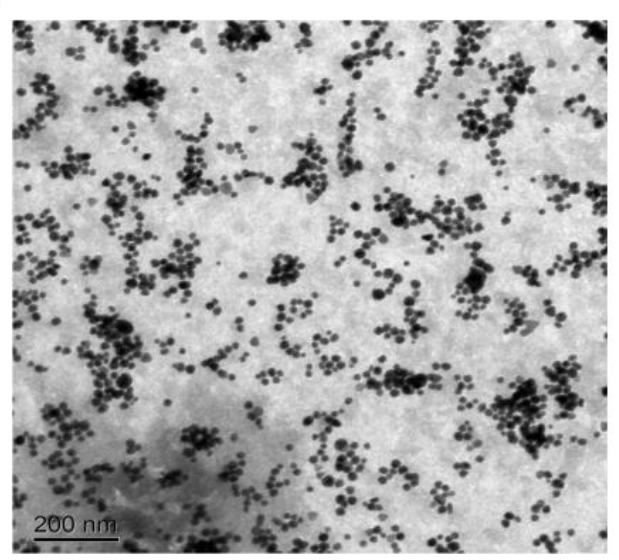

Figure S3. TEM images of collected nanostructures from the allochroic hydrogel bands which were treated with different concentrations of $\mathrm{Hg}^{2+}$ ions (a: $100 \mathrm{nM}$; b: $5 \mu \mathrm{M}$ ).

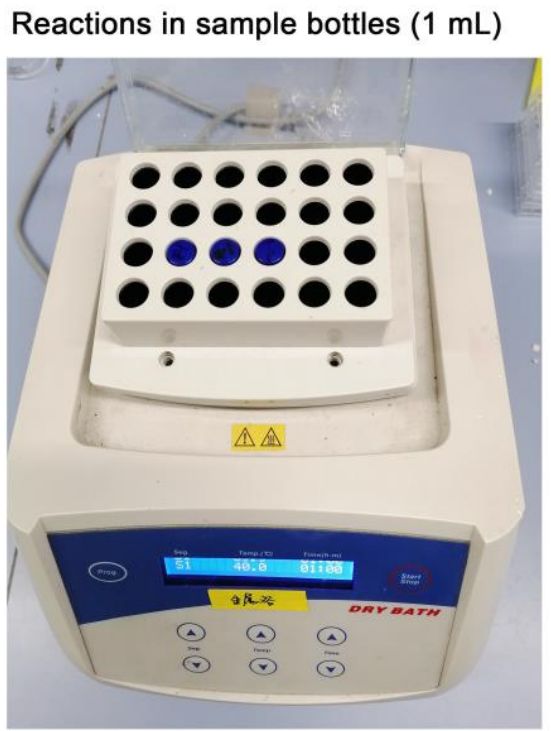

Reactions in the microplate $(100 \mu \mathrm{L})$

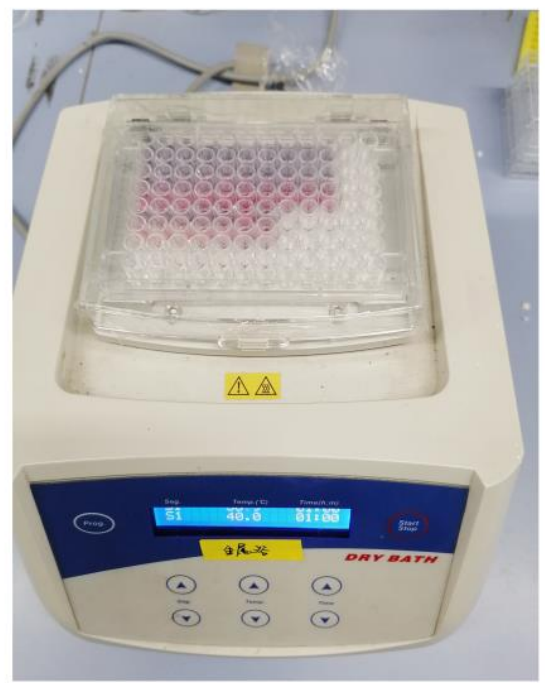

Figure S4. Based on the thermostatic metal bath, Au-staining C-AGH sensing assays $\left(40{ }^{\circ} \mathrm{C}\right)$ were conducted in sample bottles and microplates, respectively, allowing the portable measurements and high-throughput analysis. 


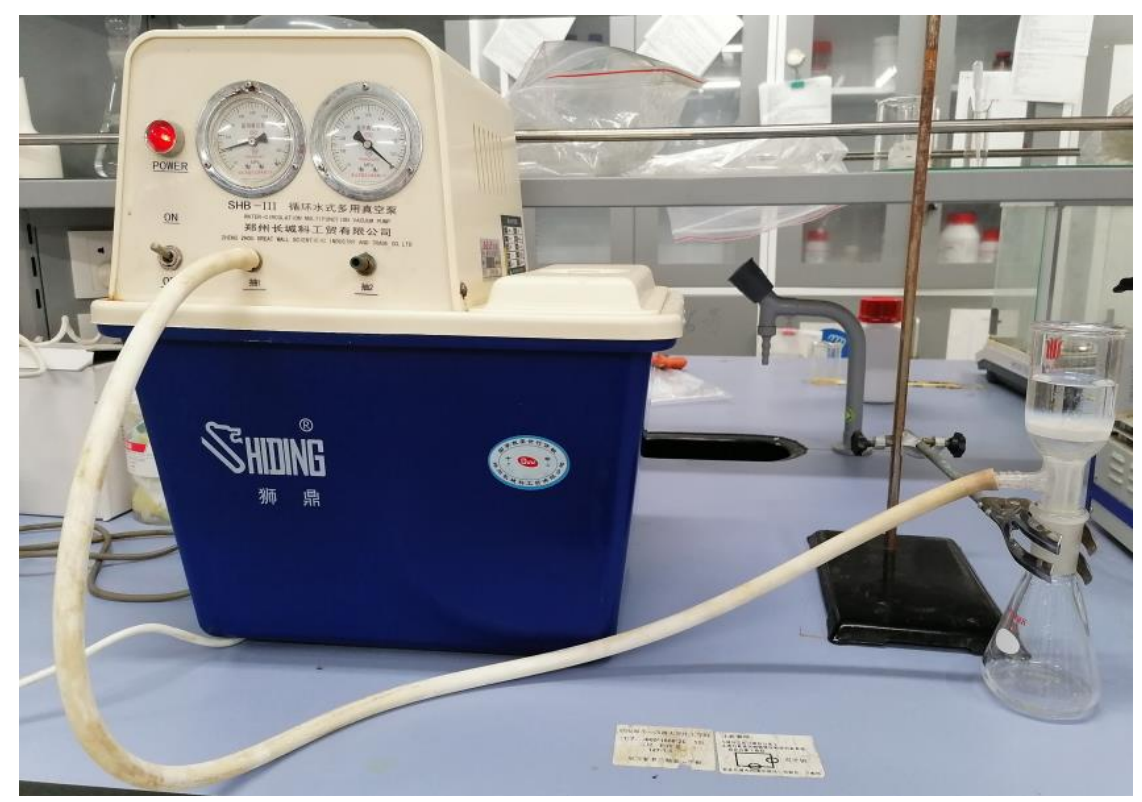

Figure S5. The apparatus showing the enrichment of $\mathrm{Hg}^{2+}$ ions using the C-AGH layer.

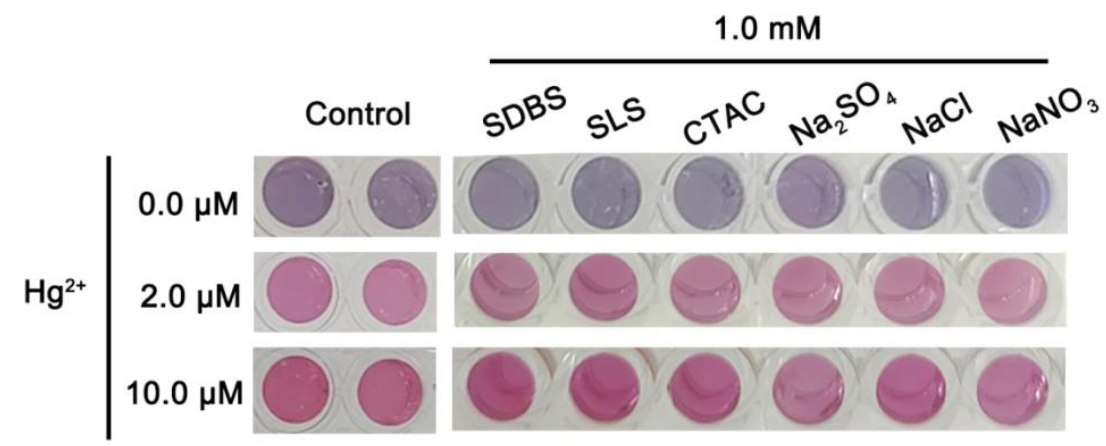

Figure S6. The portable detections of $\mathrm{Hg}^{2+}$ ions using the microplate in the presence of surfactant and anion additives including sodium dodecyl benzene sulfonate (SDBS), sodium lauryl sulfonate (SLS), hexadecyltrimethylammonium chloride (CTAC), $\mathrm{NaSO}_{4}, \mathrm{NaCl}$, and $\mathrm{NaNO}_{3}$. 
a $\quad \mathrm{Ag}^{+} \quad 0.0 \mu \mathrm{M} \quad 10.0 \mu \mathrm{M} \quad 50.0 \mu \mathrm{M} \quad 200.0 \mu \mathrm{M}$
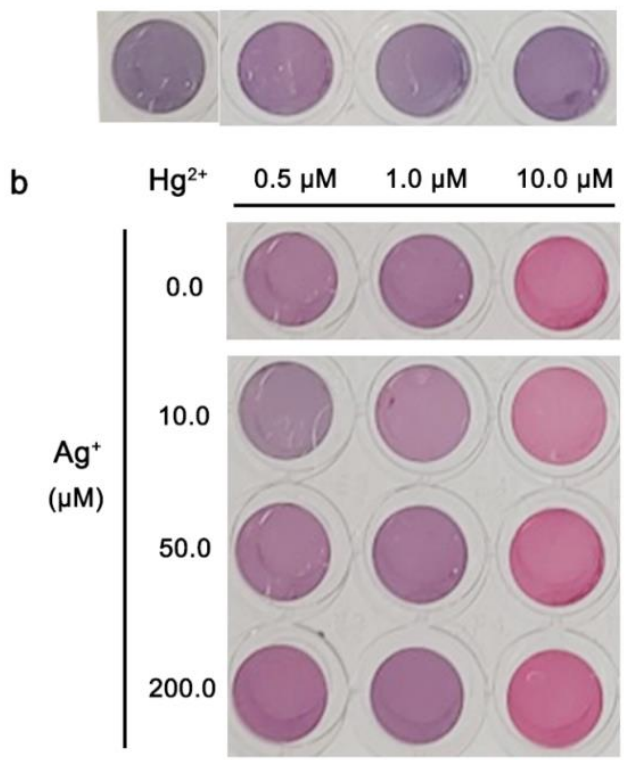

Figure S7. The responses of $\mathrm{Ag}^{+}$ions using the allochroic hydrogel sensing strategies and the influence of $\mathrm{Ag}^{+}(10.0,50.0$, and $200.0 \mu \mathrm{M})$ to the responses of $\mathrm{Hg}^{2+}$ ions with different concentrations $(0.5,1.0$, and $10.0 \mu \mathrm{M})$. 
Table S1. Comparison of Gold Nanoparticle (NP)-Based Analysis Methods for the Measurements of Mercury.

\begin{tabular}{|c|c|c|c|c|c|}
\hline Method & $\begin{array}{l}\text { Recognition } \\
\text { function }\end{array}$ & $\begin{array}{l}\text { Response } \\
\text { mechanism }\end{array}$ & $\begin{array}{l}\text { Analysis } \\
\text { substrate }\end{array}$ & $\begin{array}{l}\text { Detection } \\
\text { limit }\end{array}$ & Ref. \\
\hline $\begin{array}{l}\text { Electrochemical } \\
\text { method }\end{array}$ & $\begin{array}{l}\text { Electrochemical } \\
\text { potential }\end{array}$ & $\begin{array}{l}\text { Electrochemical } \\
\text { deposition }\end{array}$ & $\begin{array}{l}\text { Indium-tin } \\
\text { oxide electrode }\end{array}$ & $1.0 \mu \mathrm{M}$ & $\begin{array}{l}\text { Ratner } \\
\text { et al. }{ }^{[\mathrm{S} 1]}\end{array}$ \\
\hline $\begin{array}{l}\text { Electrochemical } \\
\text { method }\end{array}$ & $\begin{array}{l}\text { Thymine-rich } \\
\text { nucleic acid }\end{array}$ & $\begin{array}{l}\text { DNA } \\
\text { hybridization } \\
\text { leaded by } \mathrm{Hg}^{2+}\end{array}$ & Gold electrode & $0.5 \mathrm{nM}$ & $\begin{array}{l}\text { Zhu et } \\
a l .^{[\mathrm{S} 2]}\end{array}$ \\
\hline $\begin{array}{l}\text { Electrochemilumi } \\
\text { nescent technique }\end{array}$ & $\begin{array}{l}\mathrm{CH}_{3} \mathrm{Hg} \text {-specific } \\
\text { antigen }\end{array}$ & $\begin{array}{l}\text { The competitive } \\
\text { immunoassay }\end{array}$ & Electrode & $0.3 \mathrm{nM}$ & $\begin{array}{l}\text { Cai et } \\
a l . .^{[\mathrm{S} 3]}\end{array}$ \\
\hline $\begin{array}{l}\text { Chemiresistive } \\
\text { method }\end{array}$ & Gold NPs & $\begin{array}{l}\text { Plasmonic } \\
\text { coupling } \\
\text { influenced by } \\
\mathrm{Hg}^{2+}\end{array}$ & Transducers & $9.97 \mu \mathrm{M}$ & $\begin{array}{l}\text { McNic } \\
\text { holas et } \\
\text { al. }^{[\mathrm{S} 4]}\end{array}$ \\
\hline $\begin{array}{l}\text { Chemoresistive } \\
\text { method }\end{array}$ & $\begin{array}{l}\text { 4-Bromobenzen } \\
\text { ethiol }\end{array}$ & $\begin{array}{l}\text { Formation of } \\
\mathrm{Au}-\mathrm{Hg} \text { alloy }\end{array}$ & Transducers & $0.5 \mu \mathrm{M}$ & $\begin{array}{l}\text { Fratodd } \\
\mathrm{i} \quad \text { et } \\
a l .{ }^{[\mathrm{S} 5]}\end{array}$ \\
\hline $\begin{array}{l}\text { Field-effect } \\
\text { transistor }\end{array}$ & $\begin{array}{l}\text { Thioglycolic } \\
\text { acid }\end{array}$ & $\begin{array}{l}\mathrm{Hg}^{2+} \text { affects the } \\
\text { electron transfer }\end{array}$ & Si wafer & $25.0 \mathrm{nM}$ & $\begin{array}{l}\text { Chen et } \\
a l .{ }^{[\mathrm{S} 6]}\end{array}$ \\
\hline $\begin{array}{l}\text { Surface-enhanced } \\
\text { Raman scattering }\end{array}$ & $\begin{array}{l}\text { Thymine-rich } \\
\text { nucleic acid }\end{array}$ & $\begin{array}{l}\text { DNA } \\
\text { hybridization } \\
\text { leaded by } \mathrm{Hg}^{2+}\end{array}$ & $\begin{array}{l}\mathrm{SiO}_{2} / \mathrm{Si} \\
\text { substrate }\end{array}$ & $0.1 \mathrm{nM}$ & $\begin{array}{l}\text { Ding et } \\
a l .^{[\mathrm{S} 7]}\end{array}$ \\
\hline $\begin{array}{l}\text { Fluorescence } \\
\text { analysis }\end{array}$ & $\mathrm{Bi}^{3+}$ ions & $\begin{array}{l}\text { Catalytic } \\
\text { activities } \\
\text { declined after } \\
\text { amalgamation }\end{array}$ & Solution & $1.2 \mathrm{nM}$ & $\begin{array}{l}\text { Lien et } \\
a l .^{[\mathrm{S} 8]}\end{array}$ \\
\hline $\begin{array}{l}\text { Light-scattering } \\
\text { assay }\end{array}$ & $\begin{array}{l}\text { Thymine-rich } \\
\text { nucleic acid }\end{array}$ & $\begin{array}{l}\mathrm{Hg}^{2+} \text {-induced } \\
\text { aggregation }\end{array}$ & Solution & $1.0 \mathrm{nM}$ & $\begin{array}{l}\text { Liu et } \\
a l .{ }^{[\mathrm{S} 9]}\end{array}$ \\
\hline $\begin{array}{l}\text { Colorimetric } \\
\text { method }\end{array}$ & Lysine & $\begin{array}{l}\mathrm{Hg}^{2+} \text {-induced } \\
\text { aggregation }\end{array}$ & Solution & $2.9 \mathrm{nM}$ & $\begin{array}{l}\text { Sener } \\
e t \\
a l .{ }^{[\mathrm{S} 10]}\end{array}$ \\
\hline $\begin{array}{l}\text { Colorimetric } \\
\text { method }\end{array}$ & $\begin{array}{l}\text { Thymine-rich } \\
\text { nucleic acid }\end{array}$ & $\begin{array}{l}\mathrm{Hg}^{2+} \text {-induced } \\
\text { aggregation }\end{array}$ & $\begin{array}{l}\text { Microfluidic } \\
\text { paper }\end{array}$ & $50.0 \mathrm{nM}$ & $\begin{array}{l}\text { Chen et } \\
a l .{ }^{[\mathrm{S} 11]}\end{array}$ \\
\hline $\begin{array}{l}\text { Colorimetric } \\
\text { method }\end{array}$ & $\begin{array}{l}\text { 3-Mercaptoprop } \\
\text { ionate acid }\end{array}$ & $\begin{array}{l}\mathrm{Hg}^{2+} \text {-induced } \\
\text { aggregation }\end{array}$ & Solution & $50.0 \mathrm{nM}$ & $\begin{array}{l}\mathrm{Yu} \text { et } \\
a l .{ }^{[\mathrm{S} 12]}\end{array}$ \\
\hline
\end{tabular}




\begin{tabular}{|c|c|c|c|c|c|}
\hline $\begin{array}{l}\text { Colorimetric } \\
\text { method }\end{array}$ & $\begin{array}{l}\text { (11-mercapto-un } \\
\text { decyl)-trimethyl } \\
\text {-ammonium }\end{array}$ & $\begin{array}{l}\text { Competitive } \\
\text { conjugation } \\
\text { with the } \\
\text { stabilizers }\end{array}$ & Solution & $30.0 \mathrm{nM}$ & $\begin{array}{l}\text { Liu et } \\
a l .{ }^{[S 13]}\end{array}$ \\
\hline $\begin{array}{l}\text { Colorimetric } \\
\text { method }\end{array}$ & Gold NPs & $\begin{array}{l}\text { Catalysis of } \\
\text { gold amalgam }\end{array}$ & Solution & $1.45 \mathrm{nM}$ & $\begin{array}{l}\text { Chen et } \\
a l .{ }^{[\mathrm{S} 14]}\end{array}$ \\
\hline $\begin{array}{l}\text { Colorimetric } \\
\text { method }\end{array}$ & Cysteine & $\begin{array}{l}\text { In-situ } \\
\text { formation of } \\
\text { gold NPs }\end{array}$ & Hydrogel & $3.7 \mathrm{nM}$ & $\begin{array}{l}\text { This } \\
\text { work }\end{array}$ \\
\hline
\end{tabular}

\section{SUPPORTING REFERENCES}

[S1] Ratner, N.; Mandler, D. Electrochemical Detection of Low Concentrations of Mercury in Water Using Gold Nanoparticles. Anal. Chem. 2015, 87, 5148-5155.

[S2] Zhu, Z.; Su, Y.; Li, J.; Li, D.; Zhang, J.; Song, S.; Zhao, Y.; Li, G.; Fan, C. Highly Sensitive Electrochemical Sensor for Mercury(II) Ions by Using a Mercury-Specific Oligonucleotide Probe and Gold Nanoparticle-Based Amplification. Anal. Chem. 2009, 81, 7660-7666.

[S3] Cai, F.; Zhu, Q.; Zhao, K.; Deng, A.; Li, J. Multiple Signal Amplified Electrochemiluminescent Immunoassay for $\mathrm{Hg}^{2+}$ Using Graphene-Coupled Quantum Dots and Gold Nanoparticles-Labeled Horseradish Peroxidase. Environ. Sci. Technol. 2015, 49, 5013-5020.

[S4] McNicholas, T. P.; Zhao, K.; Yang, C.; Hernandez, S. C.; Mulchandani, A.; Myung, N. V.; Deshusses, M. A. Sensitive Detection of Elemental Mercury Vapor by Gold-Nanoparticle-Decorated Carbon Nanotube Sensors. J. Phys. Chem. C. 2011, 115, $13927-13931$.

[S5] Fratoddi, I.; Cerra, S.; Salamone, T. A.; Fioravanti, R.; Sciubba, F.; Zampetti, E.; Macagnano, A.; Generosi, A.; Paci, B.; Scaramuzzo, F. A.; Matassa, R.; Familiari, G.; Battocchio, C.; Marsotto, M.; Papa, P.; Bearzotti, A. Functionalized Gold Nanoparticles as an Active Layer for Mercury Vapor Detection at Room Temperature. ACS. Appl. Nano. Mater. 2021, 4, 2930-2940.

[S6] Chen, K.; Lu, G.; Chang, J.; Mao, S.; Yu, K.; Cui, S.; Chen, J. Hg(II) Ion Detection Using Thermally Reduced Graphene Oxide Decorated with Functionalized Gold Nanoparticles. Anal. Chem. 2012, 84, 4057-4062.

[S7] Ding, X.; Kong, L.; Wang, J.; Fang, F.; Li, D.; Liu, J. Highly Sensitive SERS Detection of $\mathrm{Hg}^{2+}$ Ions in Aqueous Media Using Gold Nanoparticles/Graphene Heterojunctions. ACS Appl. Mater. 
Interfaces 2013, 5, 7072-7078.

[S8] Lien, C-W.; Tseng, Y.-T.; Huang, C.-C.; Chang, H. T. Logic Control of Enzyme-Like Gold Nanoparticles for Selective Detection of Lead and Mercury Ions. Anal. Chem. 2014, 86, 2065-2072.

[S9] Liu, Z. D.; Li, Y. F.; Ling, J.; Huang, C. Z. A Localized Surface Plasmon Resonance Light-Scattering Assay of Mercury (II)) on the Basis of $\mathrm{Hg}^{2+}-$ DNA Complex Induced Aggregation of Gold Nanoparticles. Environ. Sci. Technol. 2009, 43, 5022-5027.

[S10] Sener, G.; Uzun, L.; Denizli, A. Lysine-Promoted Colorimetric Response of Gold Nanoparticles: A Simple Assay for Ultrasensitive Mercury(II) Detection. Anal. Chem. 2014, 86, 514-520.

[S11] Chen, G.-H.; Chen, W.-Y.; Yen, Y.-C.; Wang, C. W.; Chang, H. T.; Chen, C. F. Detection of Mercury(II) Ions Using Colorimetric Gold Nanoparticles on Paper-Based Analytical Devices. Anal. Chem. 2014, 86, 6843-6849.

[S12] Yu, C.-J.; Tseng, W.-L. Colorimetric Detection of Mercury(II) in a High-salinity Solution Using Gold Nanoparticles Capped with 3-Mercaptopropionate Acid and Adenosine Monophosphate. Langmuir 2008, 24, 12717-12722.

[S13] Liu, D.; Qu, W.; Chen, W.; Zhang, W.; Wang, Z.; Jiang, X. Highly Sensitive, Colorimetric Detection of Mercury(II) in Aqueous Media by Quaternary Ammonium Group-Capped Gold Nanoparticles at Room Temperature. Anal. Chem. 2010, 82, 9606-9610.

[S14] Chen, Z.; Zhang, C.; Gao, Q.; Wang, G.; Tan, L.; Liao, Q. Colorimetric Signal Amplification Assay for Mercury Ions Based on the Catalysis of Gold Amalgam. Anal. Chem. 2015, 87, 10963-10968. 